Acta Crystallographica Section E

Structure Reports

Online

ISSN 1600-5368

\section{myo-Inositol dihydrate: a redetermination}

\section{Arnaud Bonnet, ${ }^{a}$ William Jones ${ }^{a}$ and W. D. Samuel Motherwell ${ }^{b_{*}}$}

${ }^{\text {a }}$ The Pfizer Institute for Pharmaceutical Materials Science, Department of Chemistry, University of Cambridge, Lensfield Road, Cambridge CB2 1EW, England, and ${ }^{\mathbf{b}}$ The Cambridge Crystallographic Data Centre, 12 Union Road, Cambridge CB2 1EZ, England

Correspondence e-mail:

motherwell@ccdc.cam.ac.uk

\section{Key indicators}

Single-crystal X-ray study

$T=180 \mathrm{~K}$

Mean $\sigma(\mathrm{C}-\mathrm{C})=0.002 \AA$

$R$ factor $=0.032$

$w R$ factor $=0.101$

Data-to-parameter ratio $=13.1$

For details of how these key indicators were automatically derived from the article, see http://journals.iucr.org/e.
The crystal structure of myo-inositol dihydrate, $\mathrm{C}_{6} \mathrm{H}_{12} \mathrm{O}_{6}$-$2 \mathrm{H}_{2} \mathrm{O}$, previously reported by Lomer, Miller \& Beevers [Acta Cryst. (1963), 16, 264-268], has been redetermined, and the positions of the $\mathrm{H}$ atoms of the hydroxyl groups were located, showing an ordered hydrogen-bonding scheme.

\section{Comment}

myo-Inositol (Fig. 1) is a biological molecule of nutritional and medical importance, which has been extracted from both plant and animal sources (Posternak, 1965). The crystal structure of the anhydrous form has previously been determined (Rabinovich \& Kraut, 1964) and that of the dihydrate by Lomer et al. (1963).

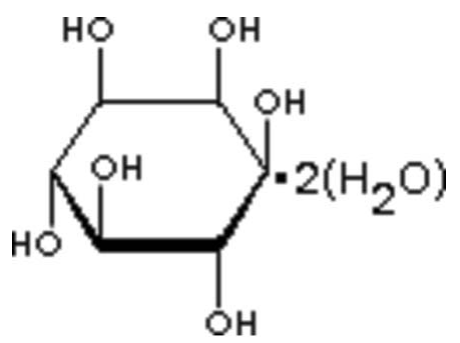

(I)

In a series of experiments aimed at inhibiting the crystallization of myo-inositol from solution, we evaporated aqueous solutions of varying concentrations of myo-inositol and polyvinylpyrrolidone. Needle-shaped crystals formed as the solutions became more concentrated at room temperature. We

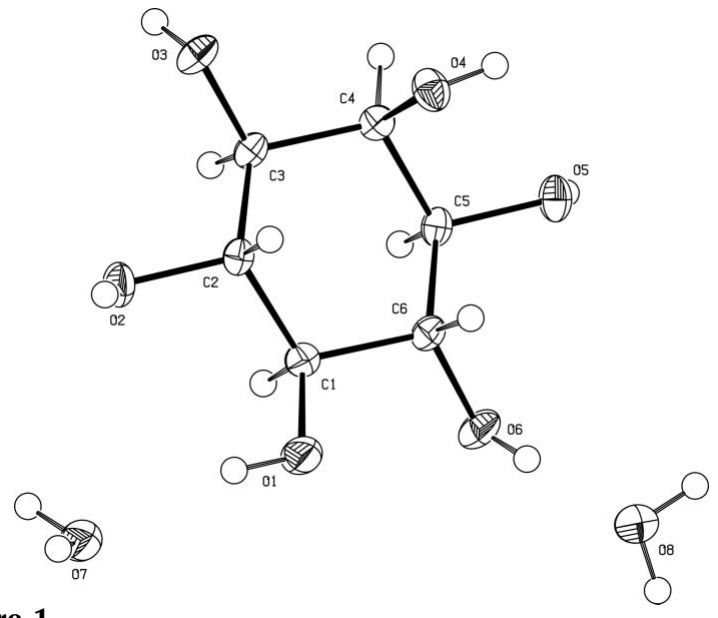

Figure 1

The aymmetric unit of myo-inositol. Displacement ellipsoids are drawn at the $50 \%$ probability level.
Received 22 May 2006 Accepted 9 June 2006
(C) 2006 International Union of Crystallography All rights reserved 


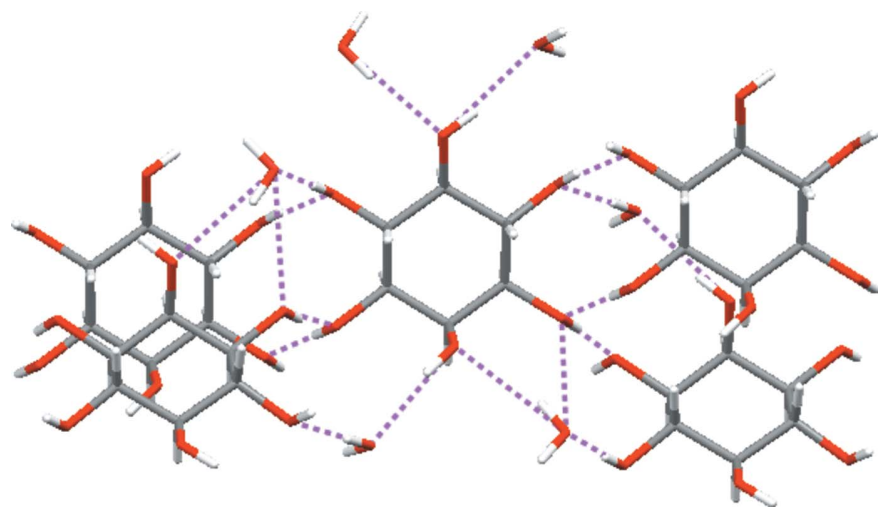

Figure 2

myo-Inositol dihydrate, showing hydrogen-bonds (dashed lines) to the neighbouring molecules.

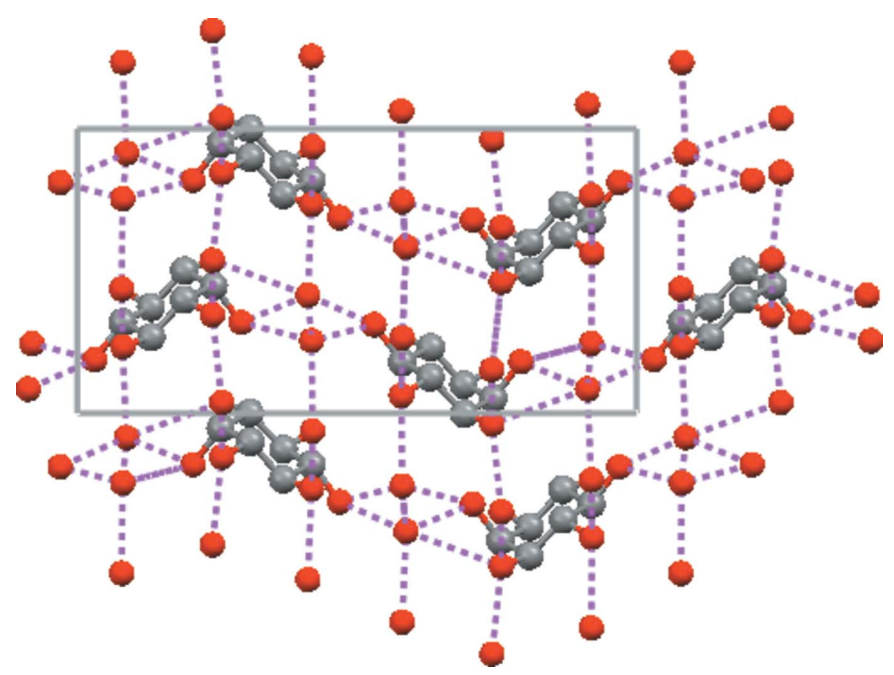

Figure 3

Packing diagram viewed in $a$ axis projection, with $b$ horizontal and $c$ vertical. $\mathrm{H}$ atoms have been omitted for clarity. Hydrogen bonds are shown as dashed lines.

obtained the structure by single-crystal X-ray diffraction analysis at $180 \mathrm{~K}$, confirming the dihydrate structure, with well located $\mathrm{H}$ atoms and a rational hydrogen-bonding scheme.

Each myo-inositol molecule forms a total of 13 intermolecular hydrogen bonds, defined as having $\mathrm{O} \cdots \mathrm{O}$ less than $3.04 \AA$ A. All 13 hydrogen bonds have normal bond lengths and geometry. The immediate hydrogen-bonded neighbours are six water molecules and four myo-inositol molecules (Fig. 2). Both water molecules show an optimal hydrogen-bond environment of two donor and two acceptor bonds. Each hydroxyl group on the inositol has a donor and acceptor hydrogen bond, with one (O3) forming (as an acceptor) a third hydrogen bond. The packing diagram (Fig. 3) shows an interesting feature where the water molecules link the inositol molecules in the $b$-axis direction, forming four-membered ring motifs $\mathrm{H}_{2} \mathrm{O} \cdots \mathrm{OH} \cdots \mathrm{H}_{2} \mathrm{O} \cdots \mathrm{OH}$.

\section{Experimental}

An aqueous solution $(10 \mathrm{ml})$ of myo-inositol $(0.426 \mathrm{~g})$ and polyvinylpyrrolidone $(0.631 \mathrm{~g})$ was prepared. The colourless solution was allowed to evaporate at room temperature. When the solution had reduced to about half its initial volume, white needle-shaped crystals were observed and analysed by single-crystal X-ray diffraction. The crystals dehydrate prior to melting at $469 \mathrm{~K}$.

\section{Crystal data}

$\mathrm{C}_{6} \mathrm{H}_{12} \mathrm{O}_{6} \cdot 2 \mathrm{H}_{2} \mathrm{O}$
$M_{r}=216.19$
Monoclinic, $P 2_{b} / n$
$a=6.6099(2) \AA$
$b=16.6009(4) \AA$
$c=9.0264(2) \AA$
$\beta=110.751(1)^{\circ}$
$V=926.22(4) \AA^{3}$

\section{Data collection}

Nonius KappaCCD diffractometer

Thin-slice $\omega$ and $\varphi$ scans

Absorption correction: multi-scan (SORTAV; Blessing, 1995)

$T_{\min }=0.905, T_{\max }=0.968$

\section{Refinement}

Refinement on $F^{2}$

$R\left[F^{2}>2 \sigma\left(F^{2}\right)\right]=0.032$

$w R\left(F^{2}\right)=0.101$

$S=1.14$

2104 reflections

161 parameters

$\mathrm{H}$ atoms treated by a mixture of independent and constrained refinement

\author{
$Z=4$ \\ $D_{x}=1.550 \mathrm{Mg} \mathrm{m}^{-3}$ \\ Mo $K \alpha$ radiation \\ $\mu=0.15 \mathrm{~mm}^{-1}$ \\ $T=180$ (2) K \\ Block cut from needle, white \\ $0.46 \times 0.35 \times 0.23 \mathrm{~mm}$
}

\section{Table 1}

Hydrogen-bond geometry $\left(\AA{ }^{\circ}{ }^{\circ}\right)$.

\begin{tabular}{|c|c|c|c|c|}
\hline$D-\mathrm{H} \cdots A$ & $D-\mathrm{H}$ & $\mathrm{H} \cdots A$ & $D \cdots A$ & $D-\mathrm{H} \cdots A$ \\
\hline $\mathrm{O} 1-\mathrm{H} 1 A \cdots \mathrm{O} 7$ & $0.82(2)$ & $2.02(2)$ & $2.8259(13)$ & $168(2)$ \\
\hline $\mathrm{O} 2-\mathrm{H} 2 A \cdots \mathrm{O} 6^{\mathrm{i}}$ & $0.81(2)$ & $1.92(2)$ & $2.7275(12)$ & $175(2)$ \\
\hline $\mathrm{O} 3-\mathrm{H} 3 A \cdots \mathrm{O} 5^{\mathrm{ii}}$ & $0.82(2)$ & $1.83(2)$ & 2.6454 (12) & $173(2)$ \\
\hline $\mathrm{O} 4-\mathrm{H} 4 A \cdots \mathrm{O} 7^{\mathrm{iii}}$ & $0.81(2)$ & $2.02(2)$ & $2.8207(12)$ & $170(2)$ \\
\hline $\mathrm{O} 5-\mathrm{H} 5 A \cdots \mathrm{O}^{\mathrm{iv}}$ & $0.84(2)$ & $1.80(2)$ & $2.6359(12)$ & $172(2)$ \\
\hline $\mathrm{O} 6-\mathrm{H} 6 A \cdots \mathrm{O} 8$ & $0.81(2)$ & $1.93(2)$ & 2.7365 & $176(2)$ \\
\hline $\mathrm{O} 7-\mathrm{H} 7 A \cdots \mathrm{O}^{\mathrm{v}}$ & $0.82(2)$ & $2.18(2)$ & 2.9903 (14) & $169(2)$ \\
\hline $\mathrm{O} 7-\mathrm{H} 7 B \cdots \mathrm{O} 2^{\mathrm{vi}}$ & $0.84(2)$ & $2.01(2)$ & $2.8442(13)$ & $179(2)$ \\
\hline $\mathrm{O} 8-\mathrm{H} 8 A \cdots \mathrm{O} 4^{\mathrm{vii}}$ & $0.82(2)$ & $2.23(2)$ & $3.0141(13)$ & $160(2)$ \\
\hline $\mathrm{O} 8-\mathrm{H} 8 A \cdots \mathrm{O} 3^{\mathrm{vii}}$ & $0.82(2)$ & $2.45(2)$ & $3.0272(13)$ & $128(2)$ \\
\hline $\mathrm{O} 8-\mathrm{H} 8 B \cdots \mathrm{O} 1^{\text {viii }}$ & $0.83(2)$ & $2.03(2)$ & 2.8525 (13) & $172(2)$ \\
\hline
\end{tabular}

Symmetry codes: (i) $x+1, y, z$; (ii) $x+\frac{1}{2},-y+\frac{1}{2}, z-\frac{1}{2}$; (iii) $-x+\frac{1}{2}, y-\frac{1}{2},-z+\frac{1}{2}$; (iv) $x-1, y, z ; \quad$ (v) $\quad-x+1,-y+1,-z+1$; (vi) $\quad-x+1,-y+1,-z$; (vii) $x-\frac{1}{2},-y+\frac{1}{2}, z+\frac{1}{2}$; (viii) $-x,-y+1,-z+1$.

All $\mathrm{OH} \mathrm{H}$ atoms were located in the final difference map without any difficulty. The positions of the $\mathrm{H}$ atoms were refined independently and successfully, with a single $\mathrm{O}-\mathrm{H}$ bond-length restraint $[\mathrm{O}-\mathrm{H}=0.807(16)-0.844(17) \AA]$ and common $U_{\text {iso }}(\mathrm{H})$ values for similar atoms $\left[U_{\text {iso }}(\mathrm{H})=0.44(2) \AA^{2}\right.$ for $\mathrm{OH}$ and $U_{\text {iso }}(\mathrm{H})=0.52(3) \AA^{2}$ for $\mathrm{H}_{2} \mathrm{O}$ ]. The remaining $\mathrm{H}$ atoms were positioned geometrically, with $\mathrm{C}-\mathrm{H}=1.00 \AA$, and refined as riding with a common displacement parameter $\left[U_{\text {iso }}(\mathrm{H})=0.206(14) \AA^{2}\right]$.

Data collection: COLLECT (Nonius, 1998); cell refinement: SCALEPACK (Otwinowski \& Minor, 1997); data reduction: SCALEPACK and DENZO (Otwinowski \& Minor, 1997); program(s) used to solve structure: SIR92 (Altomare et al., 1994); program(s) used to refine structure: SHELXL97 (Sheldrick, 1997); molecular graphics: MERCURY (Macrae et al., 2006); software used to prepare material for publication: SHELXL97. 


\section{organic papers}

The authors are grateful to Dr John Davies (Department of Chemistry, University of Cambridge) for determining the crystal structure, and Drs A. Trask and L. Fabian for help with CIF preparation. The Pfizer Institute for Pharmaceutical Materials Science is acknowledged for funding the work.

\section{References}

Altomare, A., Burla, M. C., Camalli, G., Cascarano, G., Giacovazzo, C., Guagliardi, A. \& Polidori, G. (1994). J. Appl. Cryst. 27, 435.

Blessing, R. H. (1995). Acta Cryst. A51, 33-38.
Lomer, T. R., Miller, A. \& Beevers, C. A. (1963). Acta Cryst. 16, 264 268.

Macrae, C. F., Edgington, P. R., McCabe. P., Pidcock, E., Shields, G. P., Taylor, R., Towler, M. \& van de Streek, J. (2006). J. Appl. Cryst. 39, 453-457.

Nonius, B. V. (1998). COLLECT. Nonius BV, Delft, The Netherlands.

Otwinowski, Z. \& Minor, W. (1997). Methods in Enzymology, Vol. 276, Macromolecular Crystallography, Part A, edited by C. W. Carter Jr \& R. M. Sweet, pp. 307-326. New York: Academic Press.

Posternak, T. (1965). The Cyclitols, Chemistry, Biochemisry, Biology, edited by E. Lederer, pp. 284-286. Paris: Hermann.

Rabinovich, H. N. \& Kraut, J. (1964). Acta Cryst. 17, 159-168.

Sheldrick, G. M. (1997). SHELXS97 and SHELXL97. University of Göttingen, Germany. 


\section{supporting information}

Acta Cryst. (2006). E62, o2902-o2904 [https://doi.org/10.1107/S1600536806022033]

\section{myo-Inositol dihydrate: a redetermination}

\section{Arnaud Bonnet, William Jones and W. D. Samuel Motherwell}

myo-Inositol dihydrate

\section{Crystal data}

$\mathrm{C}_{6} \mathrm{H}_{12} \mathrm{O}_{6} \cdot 2 \mathrm{H}_{2} \mathrm{O}$

$M_{r}=216.19$

Monoclinic, $P 2_{1} / n$

Hall symbol: -P $2 \mathrm{yn}$

$a=6.6099(2) \AA$

$b=16.6009(4) \AA$

$c=9.0264(2) \AA$

$\beta=110.751(1)^{\circ}$

$V=926.22(4) \AA^{3}$

$Z=4$

\section{Data collection}

Nonius KappaCCD diffractometer

Radiation source: fine-focus sealed tube thin slice $\omega$ and $\varphi$ scans

Absorption correction: multi-scan

(SORTAV; Blessing, 1995)

$T_{\min }=0.905, T_{\max }=0.968$

7754 measured reflections

\section{Refinement}

Refinement on $F^{2}$

Least-squares matrix: full

$R\left[F^{2}>2 \sigma\left(F^{2}\right)\right]=0.032$

$w R\left(F^{2}\right)=0.101$

$S=1.14$

2104 reflections

161 parameters

10 restraints

Primary atom site location: structure-invariant direct methods
$F(000)=464$

$D_{\mathrm{x}}=1.550 \mathrm{Mg} \mathrm{m}^{-3}$

Mo $K \alpha$ radiation, $\lambda=0.71073 \AA$

Cell parameters from 5712 reflections

$\theta=1.0-27.5^{\circ}$

$\mu=0.15 \mathrm{~mm}^{-1}$

$T=180 \mathrm{~K}$

Needle, white

$0.46 \times 0.35 \times 0.23 \mathrm{~mm}$

2104 independent reflections 1907 reflections with $I>2 \sigma(I)$

$R_{\text {int }}=0.019$

$\theta_{\max }=27.5^{\circ}, \theta_{\min }=2.7^{\circ}$

$h=-8 \rightarrow 8$

$k=-21 \rightarrow 21$

$l=-11 \rightarrow 11$

Secondary atom site location: difference Fourier map

Hydrogen site location: inferred from neighbouring sites

$\mathrm{H}$ atoms treated by a mixture of independent and constrained refinement

$w=1 /\left[\sigma^{2}\left(F_{\mathrm{o}}^{2}\right)+(0.0488 P)^{2}+0.2568 P\right]$

where $P=\left(F_{\mathrm{o}}{ }^{2}+2 F_{\mathrm{c}}{ }^{2}\right) / 3$

$(\Delta / \sigma)_{\max }=0.001$

$\Delta \rho_{\max }=0.27 \mathrm{e} \AA^{-3}$

$\Delta \rho_{\min }=-0.30$ e $\AA^{-3}$

Special details

Experimental. Previous report: MYTOLD in CSD.

Geometry. All e.s.d.'s (except the e.s.d. in the dihedral angle between two 1.s. planes) are estimated using the full covariance matrix. The cell e.s.d.'s are taken into account individually in the estimation of e.s.d.'s in distances, angles and torsion angles; correlations between e.s.d.'s in cell parameters are only used when they are defined by crystal symmetry. An approximate (isotropic) treatment of cell e.s.d.'s is used for estimating e.s.d.'s involving l.s. planes. 
Refinement. Refinement of $F^{2}$ against ALL reflections. The weighted $R$-factor $w R$ and goodness of fit $S$ are based on $F^{2}$, conventional $R$-factors $R$ are based on $F$, with $F$ set to zero for negative $F^{2}$. The threshold expression of $F^{2}>\sigma\left(F^{2}\right)$ is used only for calculating $R$-factors(gt) etc. and is not relevant to the choice of reflections for refinement. $R$-factors based on $F^{2}$ are statistically about twice as large as those based on $F$, and $R$ - factors based on ALL data will be even larger.

Fractional atomic coordinates and isotropic or equivalent isotropic displacement parameters $\left(\hat{A}^{2}\right)$

\begin{tabular}{lllll}
\hline & $x$ & $y$ & $z$ & $U_{\text {iso }} / U_{\text {eq }}$ \\
\hline O1 & $0.28308(15)$ & $0.46905(5)$ & $0.31146(11)$ & $0.0298(2)$ \\
H1A & $0.367(3)$ & $0.4968(11)$ & $0.285(2)$ & $0.044(2)^{*}$ \\
C1 & $0.16681(18)$ & $0.41915(6)$ & $0.18041(13)$ & $0.0186(2)$ \\
H1 & 0.0799 & 0.4544 & 0.0905 & $0.0206(14)^{*}$ \\
O2 & $0.44085(14)$ & $0.42063(5)$ & $0.06268(10)$ & $0.0227(2)$ \\
H2A & $0.563(3)$ & $0.4227(11)$ & $0.126(2)$ & $0.044(2)^{*}$ \\
C2 & $0.32068(17)$ & $0.36849(6)$ & $0.12653(12)$ & $0.0167(2)$ \\
H2 & 0.4226 & 0.3388 & 0.2194 & $0.0206(14)^{*}$ \\
O3 & $0.34860(13)$ & $0.25832(5)$ & $-0.03785(10)$ & $0.0215(2)$ \\
H3A & $0.315(3)$ & $0.2566(11)$ & $-0.135(2)$ & $0.044(2)^{*}$ \\
C3 & $0.19698(17)$ & $0.30839(6)$ & $-0.00098(12)$ & $0.0168(2)$ \\
H3 & 0.1095 & 0.3387 & -0.0984 & $0.0206(14)^{*}$ \\
O4 & $0.17370(13)$ & $0.20684(5)$ & $0.18073(10)$ & $0.0223(2)$ \\
H4A & $0.092(3)$ & $0.1746(10)$ & $0.198(2)$ & $0.044(2)^{*}$ \\
C4 & $0.04578(17)$ & $0.25630(6)$ & $0.05213(13)$ & $0.0168(2)$ \\
H4 & -0.0400 & 0.2209 & -0.0379 & $0.0206(14)^{*}$ \\
O5 & $-0.24375(14)$ & $0.25828(5)$ & $0.15365(10)$ & $0.0215(2)$ \\
H5A & $-0.370(3)$ & $0.2604(11)$ & $0.086(2)$ & $0.044(2)^{*}$ \\
C5 & $-0.10927(17)$ & $0.30913(6)$ & $0.10039(12)$ & $0.0168(2)$ \\
H5 & -0.2021 & 0.3403 & 0.0060 & $0.0206(14)^{*}$ \\
O6 & $-0.13972(14)$ & $0.41902(5)$ & $0.26532(11)$ & $0.0238(2)$ \\
H6A & $-0.119(3)$ & $0.4181(11)$ & $0.359(2)$ & $0.044(2)^{*}$ \\
C6 & $0.01239(17)$ & $0.36792(6)$ & $0.23186(12)$ & $0.0170(2)$ \\
H6 & 0.0964 & 0.3370 & 0.3294 & $0.0206(14)^{*}$ \\
O7 & $0.56987(15)$ & $0.58094(5)$ & $0.25510(12)$ & $0.0272(2)$ \\
H7A & $0.698(3)$ & $0.5847(12)$ & $0.310(2)$ & $0.052(3)^{*}$ \\
H7B & $0.566(3)$ & $0.5798(12)$ & $0.162(2)$ & $0.052(3)^{*}$ \\
O8 & $-0.05058(16)$ & $0.41219(6)$ & $0.58513(12)$ & $0.0300(2)$ \\
H8A & $-0.119(3)$ & $0.3725(10)$ & $0.595(2)$ & $0.052(3)^{*}$ \\
H8B & $-0.107(3)$ & $0.4492(13)$ & $0.620(2)$ & $0.052(3)^{*}$ \\
& & & &
\end{tabular}

Atomic displacement parameters $\left(\AA^{2}\right)$

\begin{tabular}{lllllll}
\hline & $U^{11}$ & $U^{22}$ & $U^{33}$ & $U^{12}$ & $U^{13}$ & $U^{23}$ \\
\hline O1 & $0.0305(5)$ & $0.0288(5)$ & $0.0372(5)$ & $-0.0131(4)$ & $0.0207(4)$ & $-0.0156(4)$ \\
C1 & $0.0193(5)$ & $0.0172(5)$ & $0.0210(5)$ & $-0.0006(4)$ & $0.0094(4)$ & $-0.0016(4)$ \\
O2 & $0.0193(4)$ & $0.0267(4)$ & $0.0249(4)$ & $-0.0054(3)$ & $0.0114(3)$ & $0.0009(3)$ \\
C2 & $0.0151(5)$ & $0.0192(5)$ & $0.0171(5)$ & $-0.0010(4)$ & $0.0074(4)$ & $0.0007(4)$ \\
O3 & $0.0172(4)$ & $0.0296(4)$ & $0.0192(4)$ & $0.0036(3)$ & $0.0081(3)$ & $-0.0044(3)$ \\
C3 & $0.0144(5)$ & $0.0207(5)$ & $0.0162(5)$ & $0.0019(4)$ & $0.0067(4)$ & $-0.0005(4)$
\end{tabular}




\begin{tabular}{lllllll} 
O4 & $0.0194(4)$ & $0.0205(4)$ & $0.0268(4)$ & $0.0015(3)$ & $0.0080(3)$ & $0.0057(3)$ \\
C4 & $0.0147(5)$ & $0.0190(5)$ & $0.0161(5)$ & $-0.0005(4)$ & $0.0046(4)$ & $-0.0016(4)$ \\
O5 & $0.0146(4)$ & $0.0281(4)$ & $0.0221(4)$ & $-0.0037(3)$ & $0.0070(3)$ & $0.0031(3)$ \\
C5 & $0.0134(5)$ & $0.0211(5)$ & $0.0168(5)$ & $-0.0003(4)$ & $0.0063(4)$ & $0.0018(4)$ \\
O6 & $0.0200(4)$ & $0.0290(5)$ & $0.0258(4)$ & $0.0038(3)$ & $0.0124(4)$ & $-0.0044(3)$ \\
C6 & $0.0148(5)$ & $0.0195(5)$ & $0.0184(5)$ & $0.0014(4)$ & $0.0079(4)$ & $-0.0007(4)$ \\
O7 & $0.0265(5)$ & $0.0271(5)$ & $0.0306(5)$ & $-0.0033(3)$ & $0.0134(4)$ & $-0.0051(4)$ \\
O8 & $0.0356(5)$ & $0.0245(5)$ & $0.0354(5)$ & $-0.0028(4)$ & $0.0194(4)$ & $-0.0063(4)$ \\
\hline
\end{tabular}

Geometric parameters $\left(\AA,{ }^{\circ}\right)$

\begin{tabular}{|c|c|c|c|}
\hline $\mathrm{O} 1-\mathrm{C} 1$ & $1.4255(13)$ & $\mathrm{O} 4-\mathrm{H} 4 \mathrm{~A}$ & $0.813(16)$ \\
\hline $\mathrm{O} 1-\mathrm{H} 1 \mathrm{~A}$ & $0.820(16)$ & $\mathrm{C} 4-\mathrm{C} 5$ & $1.5250(14)$ \\
\hline $\mathrm{C} 1-\mathrm{C} 6$ & $1.5221(14)$ & $\mathrm{C} 4-\mathrm{H} 4$ & 1.0000 \\
\hline $\mathrm{C} 1-\mathrm{C} 2$ & $1.5261(14)$ & $\mathrm{O} 5-\mathrm{C} 5$ & $1.4271(13)$ \\
\hline $\mathrm{C} 1-\mathrm{H} 1$ & 1.0000 & $\mathrm{O} 5-\mathrm{H} 5 \mathrm{~A}$ & $0.844(17)$ \\
\hline $\mathrm{O} 2-\mathrm{C} 2$ & $1.4271(12)$ & $\mathrm{C} 5-\mathrm{C} 6$ & $1.5267(14)$ \\
\hline $\mathrm{O} 2-\mathrm{H} 2 \mathrm{~A}$ & $0.807(16)$ & $\mathrm{C} 5-\mathrm{H} 5$ & 1.0000 \\
\hline $\mathrm{C} 2-\mathrm{C} 3$ & $1.5237(14)$ & $\mathrm{O} 6-\mathrm{C} 6$ & $1.4273(13)$ \\
\hline $\mathrm{C} 2-\mathrm{H} 2$ & 1.0000 & $\mathrm{O} 6-\mathrm{H} 6 \mathrm{~A}$ & $0.809(16)$ \\
\hline $\mathrm{O} 3-\mathrm{C} 3$ & $1.4292(13)$ & $\mathrm{C} 6-\mathrm{H} 6$ & 1.0000 \\
\hline $\mathrm{O} 3-\mathrm{H} 3 \mathrm{~A}$ & $0.824(17)$ & $\mathrm{O} 7-\mathrm{H} 7 \mathrm{~A}$ & $0.821(17)$ \\
\hline $\mathrm{C} 3-\mathrm{C} 4$ & $1.5218(14)$ & $\mathrm{O} 7-\mathrm{H} 7 \mathrm{~B}$ & $0.836(17)$ \\
\hline $\mathrm{C} 3-\mathrm{H} 3$ & 1.0000 & $\mathrm{O} 8-\mathrm{H} 8 \mathrm{~A}$ & $0.820(15)$ \\
\hline $\mathrm{O} 4-\mathrm{C} 4$ & $1.4287(13)$ & $\mathrm{O} 8-\mathrm{H} 8 \mathrm{~B}$ & $0.83(2)$ \\
\hline $\mathrm{C} 1-\mathrm{O} 1-\mathrm{H} 1 \mathrm{~A}$ & $107.8(13)$ & $\mathrm{O} 4-\mathrm{C} 4-\mathrm{C} 3$ & $108.47(8)$ \\
\hline $\mathrm{O} 1-\mathrm{C} 1-\mathrm{C} 6$ & $107.11(9)$ & $\mathrm{O} 4-\mathrm{C} 4-\mathrm{C} 5$ & $111.26(8)$ \\
\hline $\mathrm{O} 1-\mathrm{C} 1-\mathrm{C} 2$ & $111.13(9)$ & $\mathrm{C} 3-\mathrm{C} 4-\mathrm{C} 5$ & $110.24(9)$ \\
\hline $\mathrm{C} 6-\mathrm{C} 1-\mathrm{C} 2$ & $112.58(9)$ & $\mathrm{O} 4-\mathrm{C} 4-\mathrm{H} 4$ & 108.9 \\
\hline $\mathrm{O} 1-\mathrm{C} 1-\mathrm{H} 1$ & 108.6 & $\mathrm{C} 3-\mathrm{C} 4-\mathrm{H} 4$ & 108.9 \\
\hline $\mathrm{C} 6-\mathrm{C} 1-\mathrm{H} 1$ & 108.6 & $\mathrm{C} 5-\mathrm{C} 4-\mathrm{H} 4$ & 108.9 \\
\hline $\mathrm{C} 2-\mathrm{C} 1-\mathrm{H} 1$ & 108.6 & $\mathrm{C} 5-\mathrm{O} 5-\mathrm{H} 5 \mathrm{~A}$ & $107.8(13)$ \\
\hline $\mathrm{C} 2-\mathrm{O} 2-\mathrm{H} 2 \mathrm{~A}$ & $107.7(13)$ & $\mathrm{O} 5-\mathrm{C} 5-\mathrm{C} 4$ & $108.54(8)$ \\
\hline $\mathrm{O} 2-\mathrm{C} 2-\mathrm{C} 3$ & $108.58(8)$ & $\mathrm{O} 5-\mathrm{C} 5-\mathrm{C} 6$ & $109.61(8)$ \\
\hline $\mathrm{O} 2-\mathrm{C} 2-\mathrm{C} 1$ & $108.88(8)$ & $\mathrm{C} 4-\mathrm{C} 5-\mathrm{C} 6$ & $111.56(8)$ \\
\hline $\mathrm{C} 3-\mathrm{C} 2-\mathrm{C} 1$ & $111.20(8)$ & $\mathrm{O} 5-\mathrm{C} 5-\mathrm{H} 5$ & 109.0 \\
\hline $\mathrm{O} 2-\mathrm{C} 2-\mathrm{H} 2$ & 109.4 & $\mathrm{C} 4-\mathrm{C} 5-\mathrm{H} 5$ & 109.0 \\
\hline $\mathrm{C} 3-\mathrm{C} 2-\mathrm{H} 2$ & 109.4 & $\mathrm{C} 6-\mathrm{C} 5-\mathrm{H} 5$ & 109.0 \\
\hline $\mathrm{C} 1-\mathrm{C} 2-\mathrm{H} 2$ & 109.4 & $\mathrm{C} 6-\mathrm{O} 6-\mathrm{H} 6 \mathrm{~A}$ & $110.0(13)$ \\
\hline $\mathrm{C} 3-\mathrm{O} 3-\mathrm{H} 3 \mathrm{~A}$ & $108.6(13)$ & $\mathrm{O} 6-\mathrm{C} 6-\mathrm{C} 1$ & $109.34(9)$ \\
\hline $\mathrm{O} 3-\mathrm{C} 3-\mathrm{C} 4$ & $109.62(9)$ & $\mathrm{O} 6-\mathrm{C} 6-\mathrm{C} 5$ & $109.14(8)$ \\
\hline $\mathrm{O} 3-\mathrm{C} 3-\mathrm{C} 2$ & $108.89(8)$ & $\mathrm{C} 1-\mathrm{C} 6-\mathrm{C} 5$ & $110.21(8)$ \\
\hline $\mathrm{C} 4-\mathrm{C} 3-\mathrm{C} 2$ & $111.76(8)$ & $\mathrm{O} 6-\mathrm{C} 6-\mathrm{H} 6$ & 109.4 \\
\hline $\mathrm{O} 3-\mathrm{C} 3-\mathrm{H} 3$ & 108.8 & $\mathrm{C} 1-\mathrm{C} 6-\mathrm{H} 6$ & 109.4 \\
\hline $\mathrm{C} 4-\mathrm{C} 3-\mathrm{H} 3$ & 108.8 & $\mathrm{C} 5-\mathrm{C} 6-\mathrm{H} 6$ & 109.4 \\
\hline $\mathrm{C} 2-\mathrm{C} 3-\mathrm{H} 3$ & 108.8 & $\mathrm{H} 7 \mathrm{~A}-\mathrm{O} 7-\mathrm{H} 7 \mathrm{~B}$ & $105(2)$ \\
\hline $\mathrm{C} 4-\mathrm{O} 4-\mathrm{H} 4 \mathrm{~A}$ & $107.1(13)$ & $\mathrm{H} 8 \mathrm{~A}-\mathrm{O} 8-\mathrm{H} 8 \mathrm{~B}$ & $103(2)$ \\
\hline
\end{tabular}




$\begin{array}{llll}\mathrm{O} 1-\mathrm{C} 1-\mathrm{C} 2-\mathrm{O} 2 & -66.81(11) & \mathrm{O} 4-\mathrm{C} 4-\mathrm{C} 5-\mathrm{O} 5 & -57.95(11) \\ \mathrm{C} 6-\mathrm{C} 1-\mathrm{C} 2-\mathrm{O} 2 & 173.04(8) & \mathrm{C} 3-\mathrm{C} 4-\mathrm{C} 5-\mathrm{O} 5 & -178.33(8) \\ \mathrm{O} 1-\mathrm{C} 1-\mathrm{C} 2-\mathrm{C} 3 & 173.60(9) & \mathrm{O} 4-\mathrm{C} 4-\mathrm{C} 5-\mathrm{C} 6 & 62.93(11) \\ \mathrm{C} 6-\mathrm{C} 1-\mathrm{C} 2-\mathrm{C} 3 & 53.45(11) & \mathrm{C} 3-\mathrm{C} 4-\mathrm{C} 5-\mathrm{C} 6 & -57.46(11) \\ \mathrm{O} 2-\mathrm{C} 2-\mathrm{C} 3-\mathrm{O} 3 & 64.86(11) & \mathrm{O} 1-\mathrm{C} 1-\mathrm{C} 6-\mathrm{O} 6 & 63.29(11) \\ \mathrm{C} 1-\mathrm{C} 2-\mathrm{C} 3-\mathrm{O} 3 & -175.37(8) & \mathrm{C} 2-\mathrm{C} 1-\mathrm{C} 6-\mathrm{O} 6 & -174.27(8) \\ \mathrm{O} 2-\mathrm{C} 2-\mathrm{C} 3-\mathrm{C} 4 & -173.89(8) & \mathrm{O} 1-\mathrm{C} 1-\mathrm{C} 6-\mathrm{C} 5 & -176.74(8) \\ \mathrm{C} 1-\mathrm{C} 2-\mathrm{C} 3-\mathrm{C} 4 & -54.12(11) & \mathrm{C} 2-\mathrm{C} 1-\mathrm{C} 6-\mathrm{C} 5 & -54.30(11) \\ \mathrm{O} 3-\mathrm{C} 3-\mathrm{C} 4-\mathrm{O} 4 & 54.92(11) & \mathrm{O} 5-\mathrm{C} 5-\mathrm{C} 6-\mathrm{O} 6 & -63.34(11) \\ \mathrm{C} 2-\mathrm{C} 3-\mathrm{C} 4-\mathrm{O} 4 & -65.91(11) & \mathrm{C} 4-\mathrm{C} 5-\mathrm{C} 6-\mathrm{O} 6 & 176.42(8) \\ \mathrm{O} 3-\mathrm{C} 3-\mathrm{C} 4-\mathrm{C} 5 & 176.97(8) & \mathrm{O} 5-\mathrm{C} 5-\mathrm{C} 6-\mathrm{C} 1 & 176.57(8) \\ \mathrm{C} 2-\mathrm{C} 3-\mathrm{C} 4-\mathrm{C} 5 & 56.14(11) & \mathrm{C} 4-\mathrm{C} 5-\mathrm{C} 6-\mathrm{C} 1 & 56.33(11)\end{array}$

Hydrogen-bond geometry $\left(\AA,{ }^{\circ}\right)$

\begin{tabular}{lllll}
\hline$D-\mathrm{H} \cdots A$ & $D-\mathrm{H}$ & $\mathrm{H} \cdots A$ & $D \cdots A$ & $D-\mathrm{H} \cdots A$ \\
\hline $\mathrm{O} 1-\mathrm{H} 1 A \cdots \mathrm{O} 7$ & $0.82(2)$ & $2.02(2)$ & $2.8259(13)$ & $168(2)$ \\
$\mathrm{O} 2-\mathrm{H} 2 A \cdots \mathrm{O}^{\mathrm{i}}$ & $0.81(2)$ & $1.92(2)$ & $2.7275(12)$ & $175(2)$ \\
$\mathrm{O} 3-\mathrm{H} 3 A \cdots 5^{\text {ii }}$ & $0.82(2)$ & $1.83(2)$ & $2.6454(12)$ & $173(2)$ \\
$\mathrm{O} 4-\mathrm{H} 4 A \cdots 7^{\text {iii }}$ & $0.81(2)$ & $2.02(2)$ & $2.8207(12)$ & $170(2)$ \\
$\mathrm{O} 5-\mathrm{H} 5 A \cdots 3^{\text {iv }}$ & $0.84(2)$ & $1.80(2)$ & $2.6359(12)$ & $172(2)$ \\
$\mathrm{O} 6-\mathrm{H} 6 A \cdots \mathrm{O} 8$ & $0.81(2)$ & $1.93(2)$ & $2.7365(13)$ & $176(2)$ \\
$\mathrm{O} 7-\mathrm{H} 7 A \cdots \mathrm{O} 8^{\mathrm{v}}$ & $0.82(2)$ & $2.18(2)$ & $2.9903(14)$ & $169(2)$ \\
$\mathrm{O} 7-\mathrm{H} 7 B \cdots 2^{\text {vi }}$ & $0.84(2)$ & $2.01(2)$ & $2.8442(13)$ & $179(2)$ \\
$\mathrm{O} 8-\mathrm{H} 8 A \cdots{ }^{\text {vii }}$ & $0.82(2)$ & $2.23(2)$ & $3.0141(13)$ & $160(2)$ \\
$\mathrm{O} 8-\mathrm{H} 8 A \cdots{ }^{\text {vii }}$ & $0.82(2)$ & $2.45(2)$ & $3.0272(13)$ & $128(2)$ \\
$\mathrm{O} 8-\mathrm{H} 8 B \cdots{ }^{\text {vii }}$ & $0.83(2)$ & $2.03(2)$ & $2.8525(13)$ & $172(2)$
\end{tabular}

Symmetry codes: (i) $x+1, y, z$; (ii) $x+1 / 2,-y+1 / 2, z-1 / 2$; (iii) $-x+1 / 2, y-1 / 2,-z+1 / 2$; (iv) $x-1, y, z$; (v) $-x+1,-y+1,-z+1$; (vi) $-x+1,-y+1,-z$; (vii) $x-1 / 2$, $-y+1 / 2, z+1 / 2$; (viii) $-x,-y+1,-z+1$. 